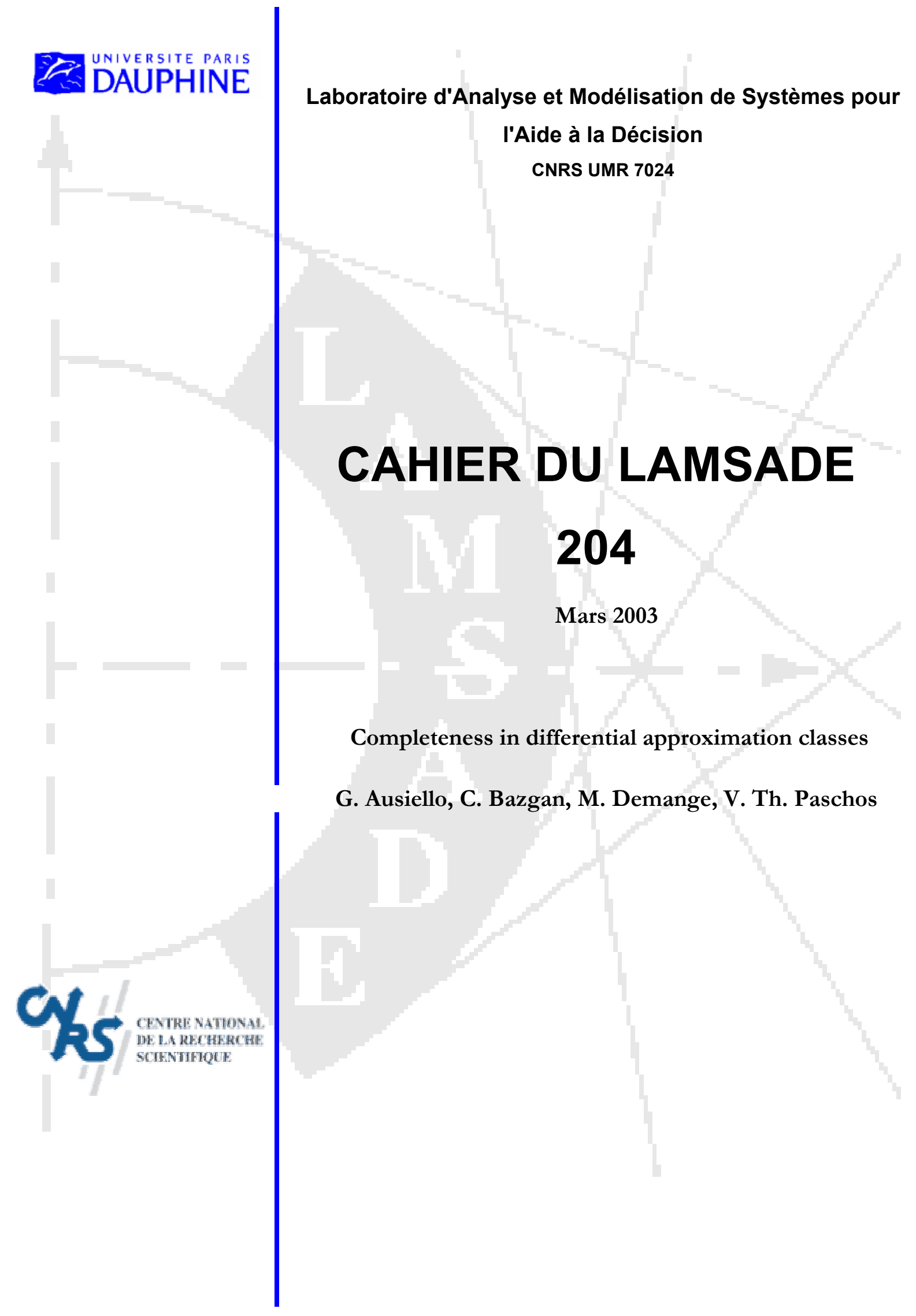




\title{
Completeness in differential approximation classes
}

\author{
Giorgio Ausiello ${ }^{1} \quad$ Cristina Bazgan $^{2} \quad$ Marc Demange $^{2,3} \quad$ Vangelis Th. Paschos ${ }^{2}$ \\ ${ }^{1}$ Dipartimento di Informatica e Sistemistica, Università degli Studi di Roma "La Sapienza" \\ via salaria 113, 00198, Roma, Italy \\ ausiello@dis. uniroma1.it \\ ${ }^{2}$ LAMSADE, Université Paris-Dauphine \\ Place du Maréchal De Lattre de Tassigny, 75775 Paris Cedex 16, France \\ \{bazgan, paschos\}@lamsade.dauphine.fr \\ ${ }^{3}$ Department of Decision and Information Systems, ESSEC, France, demange@essec.fr
}

March 13, 2003

\begin{abstract}
We study completeness in differential approximability classes. In differential approximation, the quality of an approximation algorithm is the measure of both how far is the solution computed from a worst one and how close is it to an optimal one. The main classes considered are DAPX, the differential counterpart of APX, including the NP optimization problems approximable in polynomial time within constant differential approximation ratio and the DGLO, the differential counterpart of GLO, including problems for which their local optima guarantee constant differential approximation ratio. We define natural approximation preserving reductions and prove completeness results for the class of the NP optimization problems (class NPO), as well as for DAPX and for a natural subclass of DGLO. We also define class 0-APX of the NPO problems that are not differentially approximable within any ratio strictly greater than 0 unless $\mathbf{P}=$ NP. This class is very natural for differential approximation, although has no sens for the standard one. Finally, we prove the existence of hard problems for a subclass of DPTAS, the differential counterpart of PTAS, the class of NPO problems solvable by polynomial time differential approximation schemata.
\end{abstract}

\section{Introduction}

An optimization problem $\Pi$ is in NPO if the decision version of $\Pi$ is in NP. Given an instance $x$ of $\Pi$ and a feasible solution $y$ for $x$, we denote by $\operatorname{opt}(x)$ the value of an optimal solution of $x$ and by $\omega(x)$ the value of a worst solution of $x$. In what follows, the worst-value solution $\omega(x)$ of an instance $x$ of an NPO problem $\Pi$, will be defined as the optimum solution for $x$ when seen as instance of the NPO problem $\Pi^{\prime}$, where $\Pi$ and $\Pi^{\prime}$ have the same set of instances and the same solution-set for any instance, and the goal of $\Pi^{\prime}$ is min (resp., max), if the goal of $\Pi$ is max (resp., min). Worst solutions are not always easy to compute. For instance, for the minimization version of travelling salesman problem, the worst solution is a Hamiltonian cycle of maximum total distance, i.e., the optimum solution of maximum travelling salesman problem. The computation of such a solution is not trivial since the latter problem is NP-hard. On the contrary, examples of problems for which a worst solution is easily computed are maximum independent set where the worst solution is the empty set, minimum vertex cover, or minimum graph-coloring, where the worst solution is the entire vertex-set of the input-graph.

Polynomial approximation deals with polynomial computation of "good", with respect to a predefined criterion, feasible solutions for hard NPO problems. Two main such criteria have 
been used until now: the standard approximation ratio measuring the "nearness" of a solution to an optimal one, and the differential approximation ratio measuring how a solution is ranged in the interval between a worst solution and an optimal one. More formally, for an approximation algorithm A computing a feasible solution $y$ for $x$ with value $m_{\mathrm{A}}(x, y)$, its standard approximation ratio is defined as $\gamma_{\Pi}^{\mathrm{A}}(x, y)=m_{\mathrm{A}}(x, y) / \operatorname{opt}(x)$ and its differential one as $\delta_{\Pi}^{\mathrm{A}}(x, y)=\mid \omega(x)-$ $m_{\mathrm{A}}(x, y)|/| \omega(x)-\operatorname{opt}(x) \mid$. In what follows, whenever it is understood, reference to problem $\Pi$ will be dropped. Finally note that, for any problem $\Pi$ and for any algorihm $A, 0 \leqslant \delta_{\Pi}^{\mathrm{A}} \leqslant 1$. An approximation measure $\mu$ is called cost-respecting ([22]) if given two solutions $y_{1}$ and $y_{2}$ for an instance $x$ of an optimization problem $\Pi$, the fact that $y_{1}$ is worse than $y_{2}$ implies that $\mu\left(y_{1}\right)$ is worse than $\mu\left(y_{2}\right)$. Obviously, both standard and differential approximation ratios are costrespecting measures.

Regarding the type of approximation results, NPO problems can be classified with respect to the approximation ratios known for them. So, for example, APX (DAPX) is the class of NPO problems polynomially approximable within constant (differential) ratio; PTAS (DPTAS) is the class of problems polynomially approximable by standard (differential) polynomial time approximation schemata, i.e., within standard (differential) ratios arbitrarily close to 1 ; in other words, the ratios achieved by these schemata are of the form $1-\epsilon$ (for maximization problems in standard approximation and for any problem in differential one) or $1+\epsilon$ (for minimization problems in standard approximation) for any $\epsilon>0$; finally, FPTAS (DFPTAS) is the class of problems approximable by standard (differential) fully polynomial time approximation schemata, i.e., within ratios arbitrarily close to 1 in time polynomial in both the size of their instances and in $1 / \epsilon$. Other standard approximability classes have also been defined in the litterature as, for example, LOG-APX (problems polynomially approximable within logarithmic approximation ratio), or POLY-APX (here the ratio achieved is a polynomial of the size of the instance), etc., (see, for instance, $[2,18]$ for more details about such standard approximation classes).

In this paper we study completeness for NPO, under the differential approximation ratio, for DAPX, for a subclass of DPTAS, the one of maximization problems the worst solution of which is computable in polynomial time, as well as for a subclass of DAPX, the class of problems whose local optima ensure a guaranteed differential approximation ratio with respect to their global optima.

\section{Preliminaries}

Formally, an NP optimization problem $\Pi$ is defined as a four-tuple ( $\mathcal{I}$, sol, $m$, opt) such that: $\mathcal{I}$ is the set of instances of $\Pi$ and it can be recognized in polynomial time; given $x \in \mathcal{I}$, $\operatorname{sol}(x)$ denotes the set of feasible solutions of $x$; for every $y \in \operatorname{sol}(x),|y|$ is polynomial in $|x|$; given any $x$ and any $y$ polynomial in $|x|$, one can decide in polynomial time if $y \in \operatorname{sol}(x)$; given $x \in \mathcal{I}$ and $y \in \operatorname{sol}(x), m(x, y)$ denotes the value of $y$ for $x ; m$ is polynomially computable and is commonly called feasible value, or objective value; finally, opt $\in\{\max , \min \}$. The set of $\mathbf{N P}$ optimization problems forms the class NPO. An NPO problem $\Pi$ is said to be polynomially bounded, if, for any instance $x$ of $\Pi$, the value of the optimum solution of $x$ is bounded by a polynomial in $|x|$. The set of polynomially bounded problems of NPO forms the class NPO-PB. Following the previous notations, the worst-value solution of an instance $x$ of an NPO problem $\Pi=(\mathcal{I}$, sol, $m$, opt $), \omega(x)$ is the optimum solution for $x$ with respect to the NPO problem $\Pi^{\prime}=\left(\mathcal{I}\right.$, sol, $m$, opt $\left.^{\prime}\right)$ where opt $^{\prime}=\max$, if opt $=\min$ and opt $^{\prime}=\min$, if opt $=\max$.

Since the beginning of the 80 's, researchers have been highly interested in providing a structure in standard approximation by defining suitable approximation preserving reductions in order to study completeness in approximability classes. Note that completeness for an approximability class can be seen as a way to provide lower bounds in the approximability of its members, in 
the sense that such result means that no problem of this class can have better approximability behavior unless $\mathbf{P}=\mathbf{N P}$. Pioneering works in this direction, used in this paper, are, among others, the ones in [12, 22, 23]. In [22] several natural minimization problems have been shown to be NPO-complete under an approximation preserving reduction called strict-reduction, dealing with any cost-respecting approximation measure $r$.

Definition 1. Consider two NPO problems $\Pi=(\mathcal{I}$, sol, $m$, opt $)$ and $\Pi^{\prime}=\left(\mathcal{I}^{\prime}\right.$, sol ${ }^{\prime}, m^{\prime}$, opt $)$. A strict-reduction is a pair $(f, g)$ of polynomially computable functions, $f: \mathcal{I} \rightarrow \mathcal{I}^{\prime}$ and $g$ : $\mathcal{I} \times$ sol $^{\prime} \rightarrow$ sol such that:

- $\forall x \in \mathcal{I}, x \mapsto f(x) \in \mathcal{I}^{\prime}$;

- $\forall y \in \operatorname{sol}^{\prime}(f(x)), y \mapsto g(x, y) \in \operatorname{sol}(x)$;

- if $r$ is an approximation measure, then $r_{\Pi}(x, g(x, y))$ is as good as $r_{\Pi^{\prime}}(f(x), y)$.

Throughout the paper, for any reduction $\mathrm{R}$, we will denote by $\Pi \leq_{\mathrm{R}} \Pi^{\prime}$ the fact that $\Pi \mathrm{R}$-reduces to $\Pi^{\prime}$.

In [23], the subclass MAX-SNP of APX has been introduced and complete problems have been provided for it, under L-reduction. In [12], a polynomial time approximation schema preserving reduction, called P-reduction there, has been introduced and the existence of APXcomplete problems has been shown. In what follows, we borrow the term PTAS from $[3,13]$ and we will use it instead of $P$.

Definition 2. Consider two NPO maximization problems $\Pi$ and $\Pi^{\prime}$. Then, $\Pi \leq_{\text {PTAS }} \Pi^{\prime}$ if there exist three functions $f, g$ and $c$, computable in polynomial time, such that:

- $\left.\forall x \in \mathcal{I}_{\Pi}, \forall \epsilon \in\right] 0,1\left[\cap \mathbb{Q}, f(x, \epsilon) \in \mathcal{I}_{\Pi^{\prime}}\right.$

- $\left.\forall x \in \mathcal{I}_{\Pi}, \forall \epsilon \in\right] 0,1\left[\cap \mathbb{Q}, \forall y \in \operatorname{sol}_{\Pi \Pi^{\prime}}(f(x, \epsilon)), g(x, y, \epsilon) \in \operatorname{sol}_{\Pi}(x)\right.$;

- $c:] 0,1[\cap \mathbb{Q} \rightarrow] 0,1[\cap \mathbb{Q}$;

- $\left.\forall x \in \mathcal{I}_{\Pi}, \forall \epsilon \in\right] 0,1\left[\cap \mathbb{Q}, \forall y \in \operatorname{sol}_{\Pi^{\prime}}(f(x, \epsilon)), \gamma_{\Pi^{\prime}}(f(x, \epsilon), y) \geqslant 1-c(\epsilon) \Rightarrow \gamma_{\Pi}(x, g(x, y, \epsilon)) \geqslant\right.$ $1-\epsilon$.

Furthermore, another reduction called $\mathrm{F}$ has been defined in [12] by means of which PTAScomplete problems have been provided.

Surprisingly enough, differential approximation, although introduced in [4] since 1977, has not been systematically used until the 90 's $([5,1,6,25]$ are, to our knowledge, the most notable uses of it) when a formal framework for it and a more systematic use started to be drawn $([15,16])$. In any case, no structural approach to the study of differential approximability has been developped until now. This is the main objective of this paper.

First of all, let us observe that the strict reduction in [22] is also approximation preserving with respect to differential approximation. This, as we will see in Section 3, shows the existence of NPO-complete problems also in the framework of the differential approximation. In [9], it is shown that there exist problems for which no polynomial time algorithm can guarantee that any solution computed will be even slightly far from a worst one, unless $\mathbf{P}=\mathbf{N P}$. In other words, there exist problems for which the differential ratio of any polynomial time algorithm is equal to 0 . This is the case for example for minimum independent dominating set. Such a behavior represents a worst case for the differential approximability of an NPO problem and draws a subclass of NPO called 0-DAPX in Section 3. Denote by 0-DAPX-complete, the problems of 0-DAPX sharing the following property: if one of them can be polynomially 
solved within (even non-constant) differential approximation ratio strictly greater than 0 , then all of 0-DAPX can. We prove in Section 3 that under the strict-reduction NPO-complete = 0-DAPX-complete $\subseteq \mathbf{0 - D A P X} \subseteq \mathbf{N P O}$.

In Section 4, we tackle the question of the existence of complete problems for DAPX. We define a suitable reduction, called DPTAS-reduction and show that under it many natural NPO problems are DAPX-complete.

Besides PTAS, the two most notable classes of APX in the litterature are MAX-SNP and GLO. The first one, introduced, as we have already mentioned in [23], is defined in logical terms and, furthermore, independently on any approximability property of its members; henceforth, MAX-SNP is notorious for differential approximation also without need of defining any differential counterpart for it. The latter one, GLO, is, roughly speaking, the class of the NPO-PB problems whose all locally optimal solutions (with respect to a suitable neighborhood) guarantee constant standard approximation ratio. It is introduced in [8] where a local optima preserving (LOP) reduction, which is a special case of L-reduction provided with some suitable local optimality properties, is also defined.

Definition 3. Let $\Pi^{\prime}$ and $\Pi$ be two NPO problems. An L-reduction $h=(f, g)$ is a local optima preserving reduction (LOP-reduction) if the following properties hold:

- for any $x \in \mathcal{I}_{\Pi^{\prime}}$, there exists a subset $\operatorname{sol}_{\Pi}^{\prime}(f(x)) \subseteq \operatorname{sol}_{\Pi}(f(x))$ such that:

$$
\begin{aligned}
& g\left(x, \operatorname{sol}_{\Pi}^{\prime}(f(x))\right) \supseteq \operatorname{sol}_{\Pi^{\prime}}(x) \text { (surjectivity); } \\
& \quad \forall y, z \in \operatorname{sol}_{\Pi}^{\prime}(f(x)), m_{\Pi}(f(x), y) \leqslant m_{\Pi}(f(x), z) \Rightarrow m_{\Pi^{\prime}}(x, g(x, y)) \leqslant m_{\Pi^{\prime}}(x, g(x, z)) \\
& \quad(\text { partial monotonicity); }
\end{aligned}
$$

- for every constant $k$, there exists a constant $h$ such that:

given a $k$-bounded neighborhood $N$ for $\Pi$, there exists an $h$-bounded neighborhood $N^{\prime}$ for $\Pi^{\prime}$ such that for any instance $x \in \mathcal{I}_{\Pi^{\prime}}$ and $u, z \in \operatorname{sol}^{\prime} \Pi(f(x)), u \in N(f(x), z) \Rightarrow$ $g(x, u) \in N^{\prime}(x, g(x, z))$ (locality);

$\forall z \in \operatorname{sol}_{\Pi}^{\prime}(f(x))$, if $z$ is the optimum over $\operatorname{sol}_{\Pi}^{\prime}(f(x)) \cap N(f(x), z)$, then it is the optimum over $N(f(x), z)$ (dominance).

In Section 6, we first define the differential counterpart of GLO, denoted by DGLO, as well as, two subclasses, namely $\mathbf{D G L O}_{\mathbf{0}}$, the class of maximization problems of MAX-SNP for which value 0 is a feasible one (obviously, the worst one), and $\mathbf{D G L O}_{\mathbf{A F}}$, the class of the minimization problems $\Pi$ of $\mathbf{M A X - S N P}$ for which there exists $\Pi^{\prime} \in \mathbf{D G L O}_{\mathbf{0}}$ such that $\Pi$ is affinely transformable to $\Pi$. We also devise a local optima preserving reduction strongly inspired from the LOP-reduction of [8] and, under this new reduction we prove the existence of natural complete problems for $\mathbf{D G L O}_{\mathbf{0}} \cup \mathbf{D G L O}_{\mathbf{A F}}$. Finally, by devising an appropriate reduction, we show the existence of hard problems for a natural subclass of DPTAS.

In what follows, a number of NPO problems is mentioned and/or discussed. Their definitions together with specifications of their worst solutions are given in Appendix A.

\section{Differential NPO-completeness}

We study in this section NPO-completeness with respect to differential approximation. Based upon Definition 1 of [22], given in Section 2, we define a particular strict-reduction, called Dreduction, which we use in the sequel for proving NPO-completeness. 
Definition 4. A D-reduction is a strict reduction up to the replacement of the third item in Definition 1 by the condition $\delta_{\Pi}(x, g(x, y)) \geqslant \delta_{\Pi^{\prime}}(f(x), y), \delta>0$. Two optimization problems $\Pi$ and $\Pi^{\prime}$ are D-equivalent if $\Pi$ D-reduces to $\Pi^{\prime}$ and $\Pi^{\prime}$ D-reduces to $\Pi$.

Theorem 1. MAX WSAT and MIN WSAT are D-equivalent.

Proof. We construct a differential reduction from MAX WSAT to MIN WSAT. Let $\varphi$ be an instance of MAX WSAT on $n$ variables and $m$ clauses. The instance $\varphi^{\prime}$ of MIN WSAT contains $m$ clauses and the same set of $n$ variables. With each clause $\ell_{1} \vee \ldots \vee \ell_{t}$ of $\varphi$ we associate in $\varphi^{\prime}$ the conjunction $\bar{\ell}_{1} \vee \ldots \vee \bar{\ell}_{t}$, where $\bar{\ell}_{i}=\bar{x}_{j}$ if $\ell_{i}=x_{j}$ and $\bar{\ell}_{i}=x_{j}$ if $\ell_{i}=\bar{x}_{j}$. It is easy to see that if an assignment $y$ satisfies the instance $\varphi$ then the complement of $y$ satisfies $\varphi^{\prime}$. It is easy to see that $\operatorname{opt}\left(\varphi^{\prime}\right)=\sum_{i=1}^{n} w\left(x_{i}\right)-\operatorname{opt}(\varphi)$ and $\omega\left(\varphi^{\prime}\right)=\sum_{i=1}^{n} w\left(x_{i}\right)-\omega(\varphi)$. Also, if $m\left(\varphi^{\prime}, y^{\prime}\right)$ is the value of the solution $y^{\prime}$ in $\varphi^{\prime}$, then the complement of this solution $y$ has in $\varphi$ the value $m(\varphi, y)=\sum_{i=1}^{n} w\left(x_{i}\right)-m\left(\varphi^{\prime}, y^{\prime}\right)$. Thus, $\delta(\varphi, y)=\delta\left(\varphi^{\prime}, y\right)$. The reduction from MIN WSAT to MAX WSAT is completely analogous.

As usually, ([11,22]), we denote by Max NPO and Min NPO, the classes of maximization and minimization NPO problems, respectively.

Theorem 2. MAX WSAT is Max $\mathbf{N P O}$-complete and MIN WSAT is Min $\boldsymbol{N P O}$-complete under $\leq_{\mathrm{D}}$. Max $\mathbf{N P O}$-hard and Min $\mathbf{N P O}$-hard (under $\leq_{\mathrm{D}}$ ) coincide and form the class of NPO-hard problems.

Proof. For the completeness of MIN WSAT, remark that Theorem 3.1 in [22], based upon an extension of Cook's proof ([10]) of SAT NP-completeness to optimization problems, holds for any cost-respecting approximation measure and that the differential ratio is such a measure. Furthermore, solution triv, as defined in [22] is indeed a worst-value one for MIN WSAT. The Max NPO-completeness of MAX WSAT can be proved in exactly the same way. Finally, the last assertion follows as an immediate assertion of Theorem 1.

In a completely analogous way, as in Theorem 1 one can prove the D-equivalence of MIN $\{0,1\}$ INTEger PRogramming and max $\{0,1\}$ INTEGER PROGRAMming.

Corollary 1. MIN $\{0,1\}$ INTEGER PROGRAMming and MAX $\{0,1\}$ INTEger PROGRAMming are $\mathbf{N P O}$-complete, under $\leq_{\mathrm{D}}$.

We note here that, the result of [22] about the Min NPO-completeness of MIN TSP (Theorem 3.3) can be erroneously seen as in "glaring contradiction" to a result of $[19,17]$ where it is proved that MIN TSP on graphs with polynomially bounded edge-distances is in DAPX. In fact, there is no contradiction at all. Solution triv for MIN TSP adopted in [22], is considered as a tour containing exclusively edges of maximum distance. But such a solution is not always feasible for any instance of MIN TSP (the worst-value solution for this problem is an optimal solution of MAX TSP); hence the strict reduction of Theorem 3.3 in [22] is not a D-one. Finally, solution triv adopted in [22] for MIN $\{0,1\}$ INTEGER PROGRAMMING coincides with the worst-value one as it has been adopted here in Section 1; so, the strict reduction of Theorem 3.4 in [22] is a D-one.

We now introduce an approximation class, called 0-DAPX in what follows, that seems very natural for differential approximation while has no sense in the standard case.

Definition 5. 0-DAPX is the class of NPO problems $\Pi$ for which approximation within any differential approximation ratio $\delta>0$ would entail $\mathbf{P}=\mathbf{N P}$. A problem $\Pi$ is said to be $\mathbf{0 - D A P X}$ hard, if approximation of $\Pi$ within any strictly positive differential approximation ratio would imply approximation of any other 0-DAPX problem within strictly positive approximation ratios. 
Let $\Pi^{\prime}$ be an NP-complete decision problem and $\Pi$ an NPO problem. The underlying idea for proving inclusion of $\Pi$ in 0-DAPX is, starting from an instance of $\Pi^{\prime}$, to construct instances for $\Pi$ that have only two distinct feasible values and to prove that any differential $\delta$-approximation for $\Pi^{\prime}, \delta>0$, could distinguish between positive instances and negative instances for $\Pi^{\prime}$. Intuitively, for any 0-DAPX problem, there exists at least one instance for which, unless $\mathbf{P}=\mathbf{N P}$, any polynomial time approximation algorithm cannot compute a solution other than a worst one. Remark that inclusion in 0-DAPX is rather a negative than a positive approximation result. This seems quite natural since 0-approximability represents the worst intractability level for an NPO problem in the differential approach.

Example 1. (Min indePendent Dominating SET) In [9] it is proved that if $\mathbf{P} \neq \mathbf{N P}$, then, for any decreasing $\delta: \mathbb{N} \rightarrow(0,1)$, MIN INDEPENDENT DOMINATING SET is not differential $\delta$ approximable in polynomial time. Given an instance $\varphi$ of SAT with $n$ variables $x_{1}, \ldots, x_{n}$ and $m$ clauses $C_{1}, \ldots, C_{m}$ we construct a graph $G$, instance of MIN INDEPENDENT DOMINATING SET associating with any positive literal $x_{i}$ a vertex $u_{i}$ and with any negative literal $\bar{x}_{i}$ a vertex $v_{i}$. For $i=1, \ldots, n$ we draw edges $u_{i} v_{i}$. For any clause $C_{j}$ we add in $G$ a vertex $w_{j}$ and an edge between $w_{j}$ and each vertex corresponding to a literal contained in $C_{j}$. Finally, we add edges in $G$ in order to obtain a complete graph on $w_{1}, \ldots, w_{m}$. An independent set of $G$ contains at most $n+1$ vertices since it contains at most one vertex among $w_{1}, \ldots, w_{m}$ and at most one vertex among $u_{i}$ and $v_{i}$ for $i=1, \ldots, n$. An independent dominating set containing the vertices corresponding to true literals of a non satisfiable assignment and one vertex corresponding to a clause not satisfied by this assignment is a worst solution of $G$ of size $n+1$. If $\varphi$ is satisfiable then $\operatorname{opt}(G)=n$ since the set of vertices corresponding to the true literals of an assignment satisfying $\varphi$ is an independent dominating set (each vertex $w_{j}$ is dominated by a vertex corresponding to a true literal of $C_{j}$ ) of minimum size. On the other hand, if $\varphi$ is not satisfiable then $\operatorname{opt}(G)=n+1$. So, any independent dominating set of $G$ has cardinality either $n$, or $n+1$. Hence, if an approximation algorithm achieves approximation ratio strictly greater than 0 , i.e., if it computes a solution strictly better than then worst one, then it will compute an optimal solution for $G$ (of size $n$ ), then one can correctly deduce that $\varphi$ is satisfiable, while if it returns a solution of size $n+1$, then one can correctly conclude that $\varphi$ is not satisfiable.

By analogous reductions, it is proved in [24] that for any $k>3$, polynomially bounded MAX w $k$ SAT- $B$ as well as the general minimization and maximization versions of integer-linear progamming are in 0-DAPX.

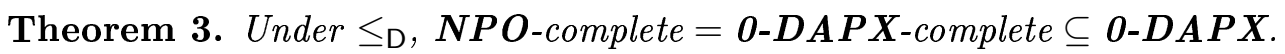

Proof. We first prove NPO-complete $\subseteq$ 0-DAPX. Fix a problem $\Pi \in$ NPO-complete. Then, for any $\Pi^{\prime} \in \mathrm{NPO}, \Pi^{\prime} \leq_{\mathrm{D}} \Pi$. Assume that MAX w $k$-SAT- $B$ stands for $\Pi^{\prime}$. Then, from [24] and by the fact MAX w $k$-SAT- $B \leq_{\mathrm{D}} \Pi$, we conclude that $\Pi \in \mathbf{0}$-DAPX.

We now prove NPO-complete $=\mathbf{0}$-DAPX-complete. Fix a problem $\Pi$ NPO-complete. For any problem $\Pi^{\prime \prime} \in \mathbf{N P O}, \Pi^{\prime \prime} \leq_{\mathrm{D}} \Pi$. Also, by the fact that NPO-complete $\subseteq \mathbf{0 - D A P X}$, $\Pi \in \mathbf{0 - D A P X}$. Since $\mathbf{0 - D A P X} \subseteq \mathbf{N P O}$, then for any problem $\Pi^{\prime} \in \mathbf{0}$-DAPX,$\Pi^{\prime} \leq_{\mathrm{D}} \Pi$ and thus $\Pi$ is $\mathbf{0 - D A P X}$-hard. In the other direction, fix a problem $\Pi \mathbf{0 - D A P X}$-complete. For any problem $\Pi^{\prime \prime} \in \mathbf{0}$-DAPX, $\Pi^{\prime \prime} \leq_{\mathrm{D}} \Pi$; in particular MAX WSAT $\leq_{\mathrm{D}} \Pi$. Since MAX WSAT is NPOcomplete, then for any $\Pi^{\prime} \in \mathbf{N P O}, \Pi^{\prime} \leq_{\mathrm{D}}$ MAX wsAT. Thus, $\Pi^{\prime} \leq_{\mathrm{D}} \Pi$ and, consequently, $\Pi$ is NPO-complete.

Finally, the inclusion 0-DAPX-complete $\subseteq$ 0-DAPX is immediate from Definition 5 .

A natural question rising from th above is: what is the relation between $\mathbf{N P O}$-complete and 0-DAPX? Taking into consideration the fact that 0-DAPXis the hardest differential approximability class in NPO, one might guass that NPO-complete $\equiv \mathbf{0 - D A P X}$, but in order 
to prove it we need a stronger reducibility. For instance, remark that Definition 5 could be stated by means of a reduction. For example, consider a kind of Turing-reduction and call it TO-reduction. Call a problem $\Pi$ TO-reducible to a problem $\Pi^{\prime}\left(\Pi \leq\right.$ TO $\left.\Pi^{\prime}\right)$, if there exists a Turing-reduction which, by addressing one or, eventually, a polynomial number of queries to an oracle for $\Pi^{\prime}$, transforms any $\delta$-differential approximation algorithm for $\Pi, \delta>0$ into an optimal (exact) algorithm for $\Pi^{\prime}$. Then, $\mathbf{0 - D A P X}$ is the class of NPO problems $\Pi$ for which there exists an NP-complete problem $\Pi^{\prime}$ such that $\Pi^{\prime} \leq$ то $\Pi$. Moreover, a problem is 0-DAPX-hard, if any problem in 0-DAPX TO-reduces to it. Note finally that TO-reduction can be seen as a particular case of both D-reduction and the strict reduction of [22]. Using then $\leq$ TO, one can prove that $\mathbf{N P O}$-complete $=\mathbf{0 - D A P X}$-complete $=\mathbf{0 - D A P X}$. In fact, since TO-reduction is in particular a D-reduction, the proof of the equality $\mathbf{N P O}$-complete $=\mathbf{0 - D A P X}$-complete in Theorem 3 always works. On the other hand, in order to prove $\mathbf{0 - D A P X}$-complete $=\mathbf{0 - D A P X}$, inclusion 0-DAPX-complete $\subseteq \mathbf{0 - D A P X}$ is immediate. For $\mathbf{0}$-DAPX-complete $\supseteq \mathbf{0}$-DAPX, we fix a 0-DAPX problem $\Pi$ and prove that any 0-DAPX-complete problem $\Pi^{\prime}$ TO-reduces to $\Pi$. Let $\Pi^{\prime \prime}$ be the NP-complete problem that has entailed the inclusion of $\Pi$ in 0-DAPX. Remark now that since $\Pi^{\prime}$ is NP-hard the optimization and decision versions of $\Pi^{\prime}$ are polynomial time equivalent ([3]); these versions are NP-hard and NP-complete and denote them by $\Pi_{O}^{\prime}$, and $\Pi_{D}^{\prime}$, respectively. The NP-completeness of $\Pi^{\prime \prime}$ and $\Pi_{D}^{\prime}$ implies $\Pi_{D}^{\prime} \leq_{\mathrm{K}} \Pi^{\prime \prime}$. On the other hand, we have $\Pi^{\prime \prime} \leq_{\mathrm{TO}} \Pi$. In all, (i) $\Pi^{\prime}=\Pi_{O}^{\prime} \equiv \Pi_{D}^{\prime} \leq_{\mathrm{K}} P \leq_{\mathrm{TO}} \Pi$, where by $\leq_{\mathrm{K}}$ we denote the usual Karp-reduction from $\Pi_{D}^{\prime}$ to $P$, and (ii) $\equiv 0 \leq_{\mathrm{K}} \circ \leq_{\mathrm{TO}}$ is a TO-reduction. So, 0-DAPX-complete $\supseteq \mathbf{0 - D A P X}$.

Figure 1 gives a summary of the class-inclusions discussed above under $\leq_{D}$. Note that problems as TSP, in both maximization and minimization versions, MAX INDEPENDENT SET, MIN COLORING, etc., are, under $\leq_{\mathrm{D}}$, NPO-intermediate, i.e., they belong to NPO $\backslash \mathbf{0}$-DAPX.

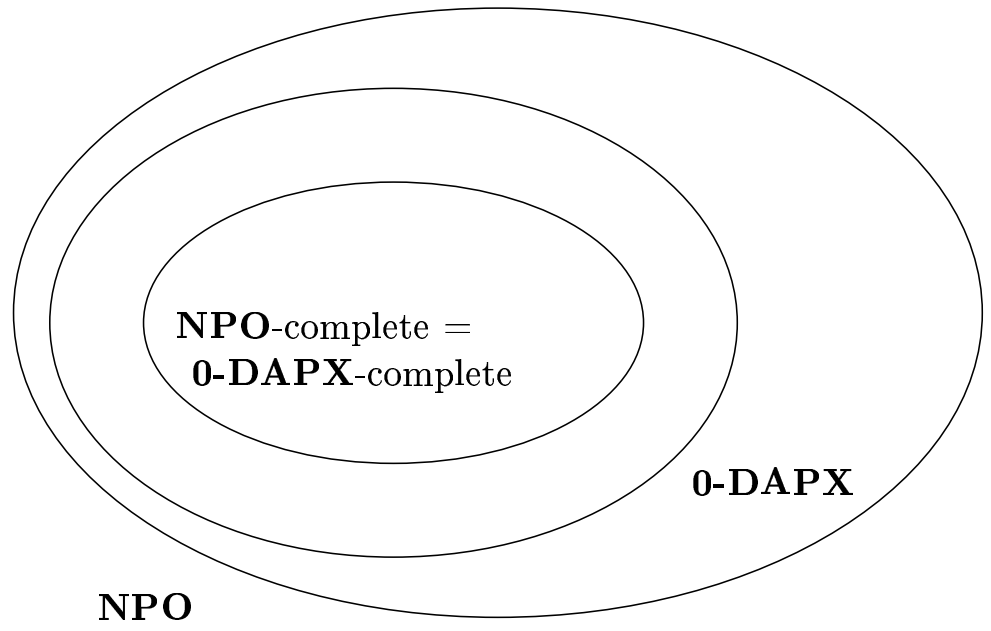

Figure 1: Class inclusions and completeness under D reduction.

\section{Differential APX-completeness}

Let us now address the problem of completeness in the class DAPX. Note first that a careful reading of the proof of the standard APX-completeness of MAX WSAT- $B$ given in [12] establishes also the following proposition which will be used in what follows.

Proposition 1. ([12]) Let $\Pi \in \boldsymbol{A P X}$. There exist 3 functions $f, g$ and $c$ such that $\forall x \in \mathcal{I}_{\Pi}$, 
$\left.\forall z \in \operatorname{sol}_{\Pi}(x), \forall \rho \in\right] 0,1[:$

1. $f(x, z, \rho)=\left(\varphi_{x, z, \rho}, W_{x, z, \rho}, w_{x, z, \rho}\right)$ with $\left(\varphi_{x, z, \rho}, w_{x, z, \rho}\right) \in \mathcal{I}_{\mathrm{MAX} \text { WSAT }} ; f$ is polynomially computable;

2. $\forall y \in \operatorname{sol}_{\operatorname{MAX}} \operatorname{WSAT}(f(x, z, \rho)), g(x, z, \rho, y) \in \operatorname{sol}_{\Pi}(x) ; g$ is polynomially computable;

3. $\left.c_{\rho}:\right] 0,1[\cap \mathbb{Q} \rightarrow] 0,1[\cap \mathbb{Q}$;

4. if $\gamma_{\Pi}(x, z) \geqslant \rho$, then $f(x, z, \rho) \in \mathcal{I}_{\text {MAX WSAT- } B}$ and, $\forall y \in \operatorname{sol}_{\operatorname{MAX}}$ WSAT- $B(f(x, z, \rho))$, if $\gamma_{\text {MAX WSAT- } B}(f(x, z, \rho), y) \geqslant 1-c_{\rho}(\epsilon)$, then $\gamma_{\Pi}(x, g(x, z, \rho, y)) \geqslant 1-\epsilon$.

Note that $f$ and $g$ are not functions of $\epsilon$ and that the reduction (the computation of $f$ ) and the interpretation (computation of $g$ ) are computable in polynomial time. The fact $z$ is a $\rho$ approximation and, consequently, that $\Pi$ is in APX, allows to consider instance $f(x, z, \rho)$ as an instance of MAX WSAT- $B$ and to consider that $g$ preserves polynomial time approximation schemata.

We first define a notion of polynomial time differential approximation schemata preserving reducibility, called DPTAS-reduction in what follows.

Definition 6. Consider two NPO problems $\Pi$ and $\Pi^{\prime}$. Then, $\Pi \leq_{\text {DPTAS }} \Pi^{\prime}$ if there exist three functions $f, g$ and $c$, computable in polynomial time, such that:

- $\left.\forall x \in \mathcal{I}_{\Pi}, \forall \epsilon \in\right] 0,1\left[\cap \mathbb{Q}, f(x, \epsilon) \in \mathcal{I}_{\Pi^{\prime}} ; f\right.$ is possibly multivalued;

- $\left.\forall x \in \mathcal{I}_{\Pi}, \forall \epsilon \in\right] 0,1\left[\cap \mathbb{Q}, \forall y \in \operatorname{sol}_{\Pi^{\prime}}(f(x, \epsilon)), g(x, y, \epsilon) \in \operatorname{sol}_{\Pi}(x)\right.$;

- $c:] 0,1[\cap \mathbb{Q} \rightarrow] 0,1[\cap \mathbb{Q}$;

- $\left.\forall x \in \mathcal{I}_{\Pi}, \forall \epsilon \in\right] 0,1\left[\cap \mathbb{Q}, \forall y \in \operatorname{sol}_{\Pi^{\prime}}(f(x, \epsilon)), \delta_{\Pi^{\prime}}(f(x, \epsilon), y) \geqslant 1-c(\epsilon) \Rightarrow \delta_{\Pi}(x, g(x, y, \epsilon)) \geqslant\right.$ $1-\epsilon$; if $f$ is multivalued, i.e., $f=\left(f_{1}, \ldots, f_{i}\right)$, for some $i$ polynomial in $|x|$, then, the former implication becomes: $\left.\forall x \in \mathcal{I}_{\Pi}, \forall \epsilon \in\right] 0,1\left[\cap \mathbb{Q}, \forall y \in \operatorname{sol}_{\Pi^{\prime}}\left(\left(f_{1}, \ldots, f_{i}\right)(x, \epsilon)\right), \exists j \leqslant i\right.$ such that $\delta_{\Pi^{\prime}}\left(f_{j}(x, \epsilon), y\right) \geqslant 1-c(\epsilon) \Rightarrow \delta_{\Pi}(x, g(x, y, \epsilon)) \geqslant 1-\epsilon$.

The following proposition can be easily shown.

Proposition 2. Given two $\boldsymbol{N P O}$ problems $\Pi$ and $\Pi^{\prime}$, if $\Pi \leq{ }_{\text {DPTAS }} \Pi^{\prime}$ and $\Pi^{\prime} \in \boldsymbol{D A P} \boldsymbol{X}$, then $\Pi \in \boldsymbol{D A P} \boldsymbol{X}$.

Let $\Pi \in \mathbf{D A P X}$ and let $\mathrm{T}$ be a differential $\rho$-approximation algorithm for $\Pi$, with $\rho \in] 0,1[$. There exists a polynomial $p$ such that $\forall x \in \mathcal{I}_{\Pi},|\omega(x)-\operatorname{opt}(x)| \leqslant 2^{p(|x|)}$. An instance $x \in \mathcal{I}_{\Pi}$ can be written in terms of an integer linear program as:

$$
x: \begin{cases}\text { opt } & v(y) \\ & y \in C_{x}\end{cases}
$$

where $C_{x}$ is the constraint-set of $x$. For any $i \in\{0, \ldots, p(|x|)\}$ and for any $l \in \mathbb{N}$, we define $x_{i, l}$ by:

- if $\Pi$ is a maximization problem, then

$$
x_{i, l}:\left\{\begin{aligned}
\max & {\left[v_{i, l}(y)=\left\lfloor\frac{v(y)}{2^{i}}\right\rfloor-l\right] } \\
y \in C_{x} &
\end{aligned}\right.
$$


- if $\Pi$ is a minimization problem, then

$$
x_{i, l}:\left\{\begin{aligned}
& \min {\left[v_{i, l}(y)=l-\left\lfloor\frac{v(y)}{2^{i}}\right\rfloor\right] } \\
& y \in C_{x}
\end{aligned}\right.
$$

Any $x_{i, l}$ can be considered as an instance of an NPO problem denoted by $\Pi_{i, l}$.

Proposition 3. Let $\epsilon<\min \{\rho, 1 / 2\}$ and $x \in \mathcal{I}_{\Pi}$. Assume $(i, l) \in\{1, \ldots, p(|x|)\} \times \mathbb{N}$ is such that $2^{i} \leqslant \epsilon|\operatorname{opt}(x)-\omega(x)| \leqslant 2^{i+1}$ and set $l=\left\lfloor\omega(x) / 2^{i}\right\rfloor$. Then, for any $y \in \operatorname{sol}_{\Pi}(x)=\operatorname{sol}_{\Pi_{i, l}}\left(x_{i, l}\right)$ :

1. $\delta_{\Pi_{i, l}}\left(x_{i, l}, y\right) \geqslant(1-\epsilon) \Longrightarrow \delta_{\Pi}(x, y) \geqslant 1-3 \epsilon$;

2. $\delta_{\Pi}(x, y) \geqslant \rho \Longrightarrow \delta_{\Pi_{i, l}}\left(x_{i, l}, y\right) \geqslant(\rho-\epsilon) /(1+\epsilon)$.

Proof. Remark that for any $(i, l)$ defined as in the statement of the proposition and $\forall y \in \operatorname{sol}_{\Pi}(x)$, $v_{i, l}(y)=\left\lfloor v(y) / 2^{i}\right\rfloor-l$, in the maximization case, and $v_{i, l}(y)=l-\left\lfloor v(y) / 2^{i}\right\rfloor$, in the minimization one. Furthermore, remark that $\delta_{\Pi}(x, y)=\delta_{\Pi_{0, l}}\left(x_{0, l}, y\right)$. For now on, we assume $\Pi$ a maximization problem (the proof in the case of minimization is similar).

In order to prove item 1 , note that

$$
\begin{aligned}
&\left\lfloor\frac{v(y)}{2^{i}}\right\rfloor-\left\lfloor\frac{\omega(x)}{2^{i}}\right\rfloor \leqslant \frac{v(y)-\omega(x)}{2^{i}}+1 \\
&\left\lfloor\frac{\operatorname{opt}(x)}{2^{i}}\right\rfloor-\left\lfloor\frac{\omega(x)}{2^{i}}\right\rfloor \geqslant \frac{\operatorname{opt}(x)-\omega(x)}{2^{i}}-1 \geqslant \frac{\operatorname{opt}(x)-\omega(x)}{2^{i}}(1-\epsilon)
\end{aligned}
$$

where (2) is true because $(\operatorname{opt}(x)-\omega(x)) / 2^{i} \geqslant 1 / \epsilon$. Combination of (1) and (2) gives $\delta_{\Pi}(x, y) \geqslant$ $(1-\epsilon)^{2}-2^{i} /(\operatorname{opt}(x)-\omega(x)) \geqslant(1-\epsilon)^{2}-\epsilon \geqslant 1-3 \epsilon$, and the proof of item 1 is complete.

For the proof of item 2 remark that

$$
\begin{aligned}
&\left\lfloor\frac{v(y)}{2^{i}}\right\rfloor-\left\lfloor\frac{\omega(x)}{2^{i}}\right\rfloor \geqslant \frac{v(y)-\omega(x)}{2^{i}}-1 \\
&\left\lfloor\frac{\operatorname{opt}(x)}{2^{i}}\right\rfloor-\left\lfloor\frac{\omega(x)}{2^{i}}\right\rfloor \leqslant \frac{\operatorname{opt}(x)-\omega(x)}{2^{i}}+1 \leqslant \frac{\operatorname{opt}(x)-\omega(x)}{2^{i}}(1+\epsilon)
\end{aligned}
$$

and combination of $(3)$ and (4) achieves $\delta_{\Pi_{i, l}}\left(x_{i, l}, y\right) \geqslant(\rho-\epsilon) /(1+\epsilon)$ that completes the proof of item 2 and of the proposition.

The proof of the existence of a DAPX-complete problem in the following Theorem 4, will be performed along the following schema. We first prove that any DAPX problem $\Pi$ is reducible to MAX WSAT- $B$ by a reduction transforming a PTAS for MAX WSAT- $B$ into a DPTAS for $\Pi$; we denote it by $\leq_{S}^{\mathrm{D}}$. Next, we consider a particular APX-complete problem $\Pi^{\prime}$, say MAX INDEPENDENT SET- $B$; MAX WSAT- $B$ that is in APX is PTAS-reducible to MAX INDEPENDENT SET- $B$. MAX INDEPENDENT SET- $B$ is both in APX and in DAPX and, moreover, standard and differential approximation ratios coincide for it; this coincidence draws a trivial reduction called ID-reduction; it trivially transforms a differential polynomial time approximation schema into a standard polynomial time approximation schema. The composition of the three reductions specified (i.e., the one from $\Pi$ to MAX WSAT- $B$, the one from MAX WSAT- $B$ to MAX INDEPENDENT SET- $B$ and the ID-reduction) is a DPTAS reduction transforming a differential polynomial time approximation schema for MAX INDEPENDENT SET- $B$ into a differential polynomial time approximation schema for $\Pi$, i.e., MAX INDEPENDENT SET- $B \in \mathbf{D A P X}$-complete. Figure 2 depicts the sketch just above.

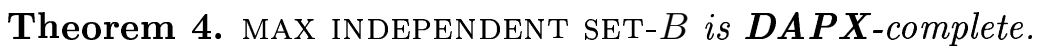




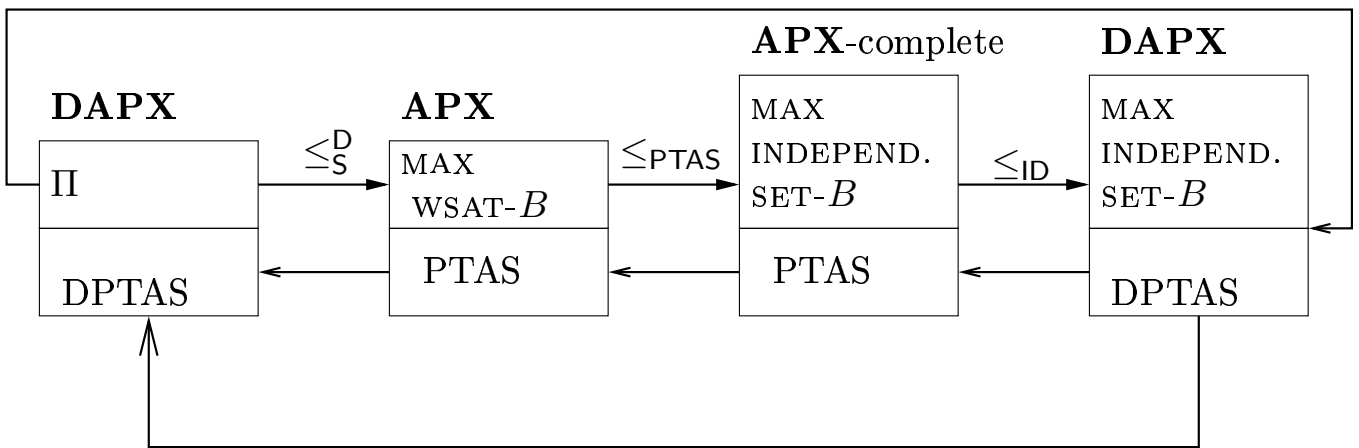

Figure 2: The schema of the proof of Theorem 4.

Proof. We first show that any integer valued problem $\Pi \in$ DAPX reduces to MAX WSAT- $B$ by a reduction transforming a standard to a differential approximation schema (extension to the case of rational values is immediate).

Remark first that given a formula $\varphi$, a variable-weight system $\vec{w}$ and a constant $B$, one can decide in polynomial time if $(\varphi, B, \vec{w}) \in \mathcal{I}_{\text {MAX WSaT- } B}$. Since $\Pi$ is in DAPX, let T be a polynomial algorithm that guarantees differential ratio $\rho \in] 0,1[$. Let be $\epsilon<\min \{\rho, 1 / 2\}$.

For any $\zeta>0$, we denote by $0_{\zeta}$ an oracle that, for any instance $x$ of MAX WSAT- $B$, computes a feasible solution $\mathrm{O}_{\zeta}(x) \in \operatorname{sol}_{\text {MAX WSAT- } B}$ guaranteeing $\gamma_{\text {MAX WSAT- } B}\left(x, 0_{\zeta}\right) \geqslant 1-\zeta$. We construct an algorithm A (reduction) using this oracle such that:

- A guarantees differential approximation ratio $1-\epsilon$ for $\Pi$ and

- in the case where $O_{\zeta}$ is polynomial (in other words, $O_{\zeta}$ can be seen as a polynomial time approximation schema), A is also polynomial.

The $\leq_{\mathrm{S}}^{\mathrm{D}}$-reduction claimed is based upon the construction of a family $\mathcal{F}$ of instances $x_{i, l}: \mathcal{F}=$ $\left\{x_{i, l}:(i, l) \in F\right\}$, where $F$ is of polynomial size and contains a pair $\left(i_{o}, l_{o}\right)$ such that:

- either $i_{0} \neq 0,2^{i_{0}} \leqslant \epsilon|\operatorname{opt}(x)-\omega(x)| \leqslant 2^{i_{0}+1}$ and $l_{0}=\left\lfloor\omega(x) / 2^{i_{0}}\right\rfloor$,

- or $i_{0}=0, \epsilon|\operatorname{opt}(x)-\omega(x)| \leqslant 2$ and $l_{0}=\omega(x)$.

Remark 1. For instance $x_{i_{0}, l_{0}}$ the worst value is 0 ; henceforth standard and differential ratios coincide. In other words, $\delta_{\Pi_{i_{0}, l_{0}}}\left(x_{i_{0}, l_{0}}, z\right)=\gamma_{\Pi_{i_{0}, l_{0}}}\left(x_{i_{0}, l_{0}}, z\right)$, for all feasible $z$.

Remark 2. For $i_{0}=0, \delta_{\Pi}(x, z)=\delta_{\Pi_{0, \omega(x)}}\left(x_{0, \omega(x)}, z\right)=\gamma_{\Pi_{0, \omega(x)}}\left(x_{0, \omega(x)}, z\right)$.

Suppose first that such a set $F$ can be constructed in polynomial time. For each $(i, l) \in F$, we consider the three functions $g_{i, l}, f_{i, l}$ and $c_{i, l}$ (Proposition 1) for the instance $x_{i, l}$. We set $\epsilon^{\prime}=\min \left\{\left(c_{i, l}\right)_{\rho}(\epsilon),\left(c_{i, l}\right)_{(\rho-\epsilon) /(1+\epsilon)}(\epsilon / 3):(i, l) \in F\right\}$ and define, for $(i, l) \in F$,

$$
\eta= \begin{cases}\rho & i=0 \\ \frac{\rho-\epsilon}{1+\epsilon} & \text { otherwise }\end{cases}
$$

Let $z=\mathrm{T}(x)$. For any $(i, l) \in F$, we set

$$
z_{i, l}= \begin{cases}g_{i, l}\left(x_{i, l}, z, \eta, 0_{\epsilon^{\prime}}\left(f_{i, l}\left(x_{i, l}, z, \eta\right)\right)\right) & \text { if } f_{i, l}\left(x_{i, l}, z, \eta\right) \text { is an instance of MAX WSAT- } B \\ z & \text { otherwise }\end{cases}
$$


Remark that $z_{i, l}$ is a feasible solution for $x_{i, l}$ and, consequently, for $x$. In all, A constructs $z_{i, l}$ for each $(i, l) \in F$ and selects the best among them as solution for $x$.

We now prove that $\mathrm{A}$ achieves differential approximation ratio $1-\epsilon$. Remark that for $(i, l)=\left(i_{0}, l_{0}\right), z=\mathrm{T}(x)$ guarantees ratio (standard or differential) $\eta$ for $x_{i_{0}, l_{0}}$ (by item 2 of Proposition 3 for the case $i_{0} \neq 0$, by Remark 2 otherwise). By item 4 of Proposition 1 applied for problem $\Pi_{i_{0}, l_{0}}$ and for $\rho=\eta$, we have: $f_{i_{0}, l_{0}}\left(x_{i_{0}, l_{0}}, z, \eta\right) \in \mathcal{I}_{\text {MAX WSAT- } B}$. Hence by de finition of $z_{i, l}, z_{i_{0}, l_{0}}=g_{i_{0}, l_{0}}\left(x_{i_{0}, l_{0}}, z, \eta, 0_{\epsilon^{\prime}}\left(f_{i_{0}, l_{0}}\left(x_{i_{0}, l_{0}}, z, \eta\right)\right)\right)$. On the other hand, recall that $\gamma_{\text {MAX WSAT- } B}\left(0_{\epsilon^{\prime}}\left(f_{i_{0}, l_{0}}\left(x_{i_{0}, l_{0}}, z, \eta\right)\right)\right) \geqslant 1-\epsilon^{\prime}$. We distinguish two cases:

- if $i_{0}=0$, then by item 4 of Proposition 1 and since $1-\epsilon^{\prime} \geqslant 1-\left(c_{0, \omega(x)}\right)_{\rho}(\epsilon)$, we have $\gamma_{\Pi_{0, \omega(x)}}\left(x_{0, \omega(x)}, z_{0, \omega(x)}\right) \geqslant 1-\epsilon$ and Remark 2 implies $\delta_{\Pi}\left(x, z_{0, \omega(x)}\right) \geqslant 1-\epsilon$;

- if $i_{0} \neq 0$, then by item 4 of Proposition 1 and since $1-\epsilon^{\prime} \geqslant 1-\left(c_{i_{0}, l_{0}}\right)_{(\rho-\epsilon) /(1+\epsilon)}(\epsilon / 3)$, we have $\gamma_{\Pi_{i_{0}, l_{0}}}\left(x_{i_{0}, l_{0}}, z_{i_{0}, l_{0}}\right) \geqslant 1-(\epsilon / 3)$ and by item 1 of Proposition 3 we have $\delta_{\Pi}\left(x, z_{i_{0}, l_{0}}\right) \geqslant 1-\epsilon$.

Since $\left(i_{0}, l_{0}\right) \in F, \mathrm{~A}$ has already computed the solution $z_{i_{0}, l_{0}}$. By taking into account that the solution finally returned by $\mathrm{A}$ is the best among the computed ones, we immediately conclude that it is at least as good as $z_{i_{0}, l_{0}}$. Therefore, it guarantees the ratio $1-\epsilon$.

We finally prove that $F$ can be constructed in polynomial time. For this, we consider two cases:

- if $\epsilon|\omega(x)-\operatorname{opt}(x)| \leqslant 2$, then $|\omega(x)-\operatorname{opt}(x)| \leqslant\lfloor 2 / \epsilon\rfloor$; in this case $\exists k \in\{-\lfloor 2 / \epsilon\rfloor, \ldots,\lfloor 2 / \epsilon\rfloor\}$ such that $\omega(x)=k+v(z)$; then $i_{0}=0$ et $l_{0}=k+v(z)$;

- if $\exists i \in\{1, \ldots p(x)\}$ such that $2^{i} \leqslant \epsilon|\operatorname{opt}(x)-\omega(x)| \leqslant 2^{i+1}$, then $\left|\left\lfloor\operatorname{opt}(x) / 2^{i}\right\rfloor-\left\lfloor\omega(x) / 2^{i}\right\rfloor\right| \leqslant$ $\lfloor 2 / \epsilon+1\rfloor ;$ so $\exists k \in\{-\lceil 2 / \epsilon+1\rceil, \ldots,\lceil 2 / \epsilon+1\rceil\}$ such that $\omega\left(x_{i, 0}\right)=\left\lfloor\omega(x) / 2^{i}\right\rfloor=v_{i, 0}(z)+k$; in this case $i_{0}=i$ and $l_{0}=v_{i, 0}(z)+k$.

Consequently, $F$ can be defined as:

$$
\begin{aligned}
F= & \left\{(0, v(z)+k): k \in\left\{-\left\lfloor\frac{2}{\epsilon}\right\rfloor, \ldots,\left\lfloor\frac{2}{\epsilon}\right\rfloor\right\}\right\} \\
& \bigcup\{1, \ldots p(x)\} \times\left\{v_{i, 0}(z)-\left\lfloor\frac{2}{\epsilon}+1\right\rfloor, \ldots, v_{i, 0}(z)+\left\lfloor\frac{2}{\epsilon}+1\right\rfloor\right\} .
\end{aligned}
$$

Remark that since $F$ is of polynomial cardinality, the number of oracle's calls is also polynomial. So the total complexity is polynomial. For simplicity, we simply use $\leq$ to denote the reduction just exhibited; in all we have shown that $\forall \Pi \in \mathbf{D A P X}$,

$$
\Pi \leq_{\mathrm{S}}^{\mathrm{D}} \operatorname{MAX} \text { WSAT- } B
$$

and the transformation of a PTAS for MAX WSAT- $B$ into a DPTAS for any problem $\Pi \in$ DAPX is complete.

Since maX indePendent SET- $B$ is APX-complete, any problem in APX, a fortiori maX WSAT- $B$, reduces to MAX INDEPENDENT SET- $B$, i.e.,

$$
\text { MAX WSAT- } B \leq \text { PTAS MAX INDEPENDENT SET- } B
$$

On the other hand, since, for any instance $G$ of MAX InDEPENDENT SET, $\omega(G)=0$, standard and differential approximation ratios coincide; hence,

$$
\text { MAX INDEPENDENT SET- } B \leq \text { ID MAX INDEPENDENT SET- } B
$$


The composition of reductions in (5), (6) and (7), i.e., $\leq_{\mathrm{S}}^{\mathrm{D}} \circ \leq_{\mathrm{PTAS}} \circ \leq_{\mathrm{ID}}$ clearly fits Definition 6 ; therefore, this composition is a DPTAS-reduction.

In all, we have shown that, $\forall \Pi \in$ DAPX, $\Pi \leq$ DPTAS MAX INDEPENDENT SET- $B$. Furthermore, MAX INDEPENDENT SET- $B$ is in DAPX, since any algorithm computing a maximal (for the inclusion) independent set guarantees a differential approximation ratio $1 /(B+1)$ (recall that $\omega(G)=0$ for any $G$ ). Consequently, MAX INDEPENDENT SET- $B$ is DAPX-complete and the proof of the theorem is complete.

Theorem 5. min vertex cover- $B$, max set paCking- $B$, min Set COVer- $B$, are $\boldsymbol{D} \boldsymbol{A} \boldsymbol{P} \boldsymbol{X}$ complete under DPTAS-reductions.

Proof. Note first that MAX Set PACKIng- $B$, Min Vertex COVER- $B$ and Min SET COver- $B$ belong to DAPX. As for MAX INDEPENDENT SET- $B$, any approximation algorithm computing a maximal (for the inclusion) set packing achieves differential approximation ratio $1 / B$ (recall that the worst-value solution for MAX SET PACKING- $B$ is the empty set; so, standard and differential approximation ratios coincide). MIN VERTEX COVER is equivalent to MAX INDEPENDENT SET via affine transformation of their objective functions and the differential approximation ratio is stable for such transformations; henceforth, MIN VERTEX COVER- $B$ is approximable within differential approximation ratio $1 / B$. Finally, the proof of the inclusion of MIN SET COVER- $B$ in DAPX follows from $[14,16]$ where in [16] it is proved that when $|C| \leqslant|\mathcal{S}|$, then MIN SET COVER is approximable within differential approximation ratio $1 / 2$, while in [14] it is proved that for $|C| \geqslant|\mathcal{S}|$, MIN SET COVER- $B$ is approximable within differential ratio $1 / B$.

The DAPX-hardness for MIN VERTEX COVER is immediate. On the other hand, MAX SET PACKING, is approximate equivalent for both standard and differential approximations under reductions preserving constant approximation ratios (as well as ratios depending on the order of the input-graph) to MAX INDEPENDENT SET.

The hardness of MIN SET COVER- $B$ for DAPX can be proved by the remark that MIN VERTEX COVER- $B$ is the restriction of MIN SET COVER- $B$ in set systems where any element of the ground set $C$ belongs to exactly two sets of the family $\mathcal{S}$ (considering a graph as a set-system where any vertex is a set containing its adjacent edges).

Theorem 6. MAX INDEPENDENT SET, MIN VERTEX COVER, MAX SET PACKING, MIN SET COVER, MAX CLIQUE and MAX $\ell$-COLORABLE INDUCED SUBGRAPH, are $\boldsymbol{D A P} \boldsymbol{X}$-hard under DPTAS-reductions.

Proof. The hardness of the first four problems is immediate from Theorem 5. The DAPXhardness for MAX CLIQUE comes from the fact that MAX INDEPENDENT SET and MAX CLIQUE are approximate equivalent for both standard and differential approximations under reductions preserving constant approximation ratios (as well as ratios depending on the order of the inputgraph).

For the hardness of MAX $\ell$-COLORABLE INDUCED SUBGRAPH consider a graph $G(V, E)$, instance of MAX INDEPENDENT SET and consider graph $G_{\ell}\left(V_{\ell}, E_{\ell}\right)$ consisting of $\ell$ copies of $G$, any two distinct copies being linked completely. Consider $B_{\ell}$ a solution for MAX $\ell$-COLORABLE INDUCED SUBGRAPH on $G_{\ell}$ and denote by $V_{\ell}^{\prime}$ its vertex-set. Obviously, the greatest among the colors in $V_{\ell}^{\prime}$, denoted by $V^{\prime}$, is an independent set of $G$; hence $m\left(G, V^{\prime}\right) \geqslant m\left(G_{\ell}, V_{\ell}^{\prime}\right) / \ell$. On the other hand, given a maximum independent set of $G$ the solution consisting of taking one copy of it in each of the $\ell$ copies of $G$ in $G_{\ell}$ is feasible for MAX $\ell$-COLORABLE INDUCED SUBGRAPH; so, $\operatorname{opt}\left(G_{\ell}\right) \geqslant \ell \operatorname{opt}(G)$. Combining expressions above and taking into account that for both problems worst-values are 0 , one concludes that the reduction just described is a DPTAS-one. I

Let us note that if we restrict ourselves in the class of DAPX problems with $\omega(x)$ computable in polynomial time, denoted by $\mathbf{D A P X} \mathbf{p}$, then the proof of the existence of $\mathbf{D A P X} \mathbf{X}_{\mathbf{p}}$-complete 
problems can be much simplified. In fact, consider an instance $x$ of such a problem $\Pi$ (assume without loss of generality that $\Pi$ is a maximization problem) and an instance $x^{\prime}$ of problem $\mathcal{P}^{\prime}$ which is identical to $\Pi$, i.e., both problems are defined on the same inputs and have identical sets of constraints; consequently they have the same set of solutions. Consider finally that $m_{\Pi^{\prime}}\left(x^{\prime}, y\right)=m_{\Pi}(x, y)-\omega(x)$. Since differential ratio is stable under affine transformations of the objective function, if $\Pi \in \mathbf{D A P X}$, then $\Pi^{\prime} \in \mathbf{D A P X}$. Moreover, it is easy to see that $\delta_{\Pi}(x, y)=\delta_{\Pi^{\prime}}\left(x^{\prime}, y\right)=\gamma_{\Pi^{\prime}}\left(x^{\prime}, y\right)$; in other words $\Pi^{\prime}$ belongs to both DAPX and APX. We so have: $\Pi \equiv_{\mathrm{D}} \Pi^{\prime} \leq$ PTAS MAX INDEPENDENT SET- $B$, where $\equiv_{\mathrm{D}}$ denotes the differential approximation equivalence between $\Pi$ and $\Pi^{\prime}$; since this later problem is in $\mathbf{D A P X}_{\mathbf{p}}$ and $\equiv_{\mathrm{D}}$ $\circ \leq$ PTAS is a DPTAS-reduction, we get that MAX INDEPENDENT SET- $B$ is DAPX $\mathbf{p}_{\mathbf{p}}$-complete. The additional complexity of the proof of Theorem 4 is for taking into account problems for which worst solution is not polynomially computable.

\section{Differential PTAS-hardness}

In this section, we will take into consideration the class DPTAS and we will address the problem of completeness in such class.

Consider the following reduction preserving fully polynomial time differential approximation schemata, denoted by DFPTAS-reduction in what follows.

Definition 7. Assume two NPO problems $\Pi$ and $\Pi^{\prime}$. Then, $\Pi \leq_{\text {DFPTAS }} \Pi^{\prime}$, if there exist three functions $f, g$ and $c$ such that:

- $f$ and $g$ are as in Definition 2;

- $\left.c:(] 0,1[\cap \mathbb{Q}) \times \mathcal{I}_{\Pi} \rightarrow\right] 0,1[\cap \mathbb{Q}$; its time complexity and its value are polynomial in both $|x|$ and $1 / \epsilon$;

- $\left.\forall x \in \mathcal{I}_{\Pi}, \forall \epsilon \in\right] 0,1\left[\cap \mathbb{Q}, \forall y \in \operatorname{sol}_{\Pi^{\prime}}(f(x, \epsilon)), \delta_{\Pi^{\prime}}(f(x, \epsilon), y) \geqslant 1-c(\epsilon, x) \Rightarrow \delta_{\Pi}(x, g(x, y, \epsilon)) \geqslant\right.$ $1-\epsilon$.

Proposition 4. Given two NPO problems $\Pi$ and $\Pi^{\prime}$, if $\Pi \leq_{\text {DFPTAS }} \Pi^{\prime}$ and $\Pi^{\prime} \in \boldsymbol{D P T A S}$, then $\Pi \in \boldsymbol{D P T A S}$.

In the following we will apply the simplification drawn at the end of Section 4, in order to study completeness, not for the whole class DPTAS but for a subclass DPTAS $_{\mathbf{p}}$ consisting of the maximization problems of PTAS the worst-value of which is computable in polynomial time (this class includes, in particular, maximization problems with worst value 0 ). Recall that, the first problem proved PTAS-complete (under FPTAS reduction) is MAX LINEAR WSAT- $B$ ([12]).

Consider a problem $\Pi \in \mathbf{D P T A S}_{\mathbf{p}}$ and an instance $x \in \mathcal{I}_{\Pi}$ expressed, in terms of an integer linear program as:

$$
x:\left\{\begin{array}{c}
\operatorname{opt} v(y) \\
y \in C_{x}
\end{array}\right.
$$

Consider also a new problem $\Pi^{\prime}$ and an instance $x^{\prime} \in \mathcal{I}_{\Pi^{\prime}}$ whose integer-linear programming formulation is

$$
x^{\prime}:\left\{\begin{array}{c}
\operatorname{opt} v\left(y^{\prime}\right)-\omega(x) \\
y^{\prime} \in C_{x^{\prime}} \\
C_{x}=C_{x^{\prime}}
\end{array}\right.
$$

Obviously, $y=y^{\prime}$, consequently, $\operatorname{sol}_{\Pi}(x)=\operatorname{sol}_{\Pi^{\prime}}\left(x^{\prime}\right), \Pi^{\prime} \in \mathbf{D P T A S}_{\mathbf{p}}$ (more precisely, for any $\left.x^{\prime} \in \mathcal{I}_{\Pi^{\prime}}, \omega\left(x^{\prime}\right)=0\right)$ and,

$$
\delta_{\Pi}(x, y)=\delta_{\Pi^{\prime}}\left(x^{\prime}, y^{\prime}\right)=\gamma_{\Pi^{\prime}}\left(x^{\prime}, y^{\prime}\right)
$$


Furthermore, since $\Pi \in \mathbf{D P T A S}_{\mathbf{p}}$, so does $\Pi^{\prime}$ (the differential ratio is stable under affine transformations of the objective function). On the other hand, by (8), $\Pi^{\prime} \in \mathbf{P T A S}$; hence $\Pi^{\prime} \leq$ FPTAS MAX LiNEAR WSAT- $B$. In all, for any $\Pi \in$ DPTA $_{\mathbf{p}}, \Pi \equiv_{\mathrm{D}} \Pi^{\prime} \leq$ FPTAS MAX LinEAR WSAT- $B$, where $\Pi^{\prime}$ is defined as above and $\equiv_{D}$ denotes, as previously, the differential approximation equivalence between $\Pi$ and $\Pi^{\prime}$. Obviously, reduction $\equiv_{\mathrm{D}} \circ \leq_{\text {FPTAS }}$ is a DFPTAS-reduction.

Consider now the closure of DPTAS $\mathbf{p}$ under affine transformations of objective functions of its problems:

$$
{\overline{\text { DPTAS }_{\mathbf{p}}}}^{\mathbf{A F}}=\left\{\Pi \in \mathbf{D P T A S}: \exists \Pi^{\prime} \in \mathbf{D P T A S}_{\mathbf{p}}, \Pi^{\prime} \leq \mathrm{AF} \Pi\right\}
$$

Let us note that $\overline{\mathbf{D P T A S}}_{\mathbf{p}}$ AF does not coincide with DPTAS $_{\mathbf{p}}$. Indeed, MAX INDEPENDENT SET in planar graphs, being in PTAS and having worst value 0 , is in DPTAS $_{\mathbf{p}}$; henceforth, MIN VERTEX COVER in planar graphs is in $\overline{\operatorname{DPTAS}}_{\mathbf{p}} \mathbf{A F}$.

Let any $\Pi^{\prime \prime} \in{\overline{\text { DPTAS }_{\mathbf{p}}}}^{\mathbf{A F}}$ and $\Pi$ its "affine mate" in $\mathbf{D P T A S}_{\mathbf{p}}$. Then, $\Pi$ " $\leq_{\mathrm{AF}} \Pi \equiv_{\mathrm{D}}$

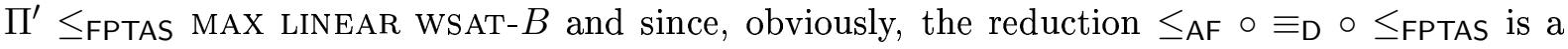
DFPTAS-one, the following proposition holds.

Proposition 5. MAX LINEAR WSAT- $B$ is ${\overline{\text { DPTAS }_{\mathbf{p}}}}^{\text {AF }}$-hard, under $\leq_{\text {DFPTAS }}$.

\section{MAX-SNP and differential GLO}

In the theory of approximability of optimization problems based upon the standard approximation ratio interesting results have been obtained by studying the behavior of local search heuristics and the degree of approximation that such heuristics can achieve. In particular, in [8,7], the class GLO is defined as the class of polynomially bounded optimization problems whose local optima have a guaranteed quality with respect to the global optima. More precisely, given an NPO problem $\Pi$, a neighborhood is a function $N: \mathcal{I}_{\Pi} \times \operatorname{sol}\left(\mathcal{I}_{\Pi}\right) \rightarrow 2^{\operatorname{sol}\left(\mathcal{I}_{\Pi}\right)}$, computable in polynomial time, such that for any $x \in \mathcal{I}_{\Pi}$ and $y \in \operatorname{sol}(x), N(x, y) \subseteq \operatorname{sol}(x)$. Solution $y$ is a local optimum of $x$ w.r.t. $N$ if for every $y^{\prime} \in N(x, y), m(x, y)$ is better than $m\left(x, y^{\prime}\right)$. Problem $\Pi$ has guaranteed local optima, if there exists a neighborhood $N$ and a constant $r$ such that for any $x \in \mathcal{I}_{\Pi}$ any local optimum $y$ for $x$ guarantees standard approximation ratio $r$. Given $y$ and $y^{\prime}$ in $\operatorname{sol}(x), y^{\prime}$ is an $h$-bounded neighbor of $y$ if it is obtained from $y$ by changing at most $h$ elements of it. A neighborhood $N$ is called $h$-bounded if there exists an integer constant $h$, such that any $y^{\prime} \in N(x, y)$ is an $h$-bounded neighbor of $y$. Based upon the above concepts the class GLO is defined as follows.

Definition 8. Let $\Pi$ be a polynomially bounded NPO problem. Then, $\Pi \in$ GLO if (i) at least one feasible $y \in \operatorname{sol}(x)$ can be computed in polynomial time, for every $x \in \mathcal{I}_{\Pi}$ and (ii) there exist an integer $h \in \mathbb{N}$ and a suitable $h$-bounded neighborhood $N$ such that $\Pi$ has guaranteed local optima with respect to $N$.

Of course, the differential counterpart of GLO, called DGLO in what follows, can be defined analogously. In [20] it is shown that MAX CUT, MIN DOMINATING SET- $B$, MAX INDEPENDENT SET- $B$, MIN VERTEX COVER- $B$, MAX SET PACKING- $B$, MIN COLORING, MIN SET COVER- $B$ MIN SET $\mathrm{W}(K)$ COVER- $B$, MIN FEEDBACK EDGE SET, MIN FEEDBACK VERTEX SET- $B$ and MIN MUltiprocessor SCHEDUling, are included in DGLO. Furthermore in [21] it is proved that both MIN and MAX TSP on graphs with polynomially bounded edge-distances are also included in DGLO. In the opposite, as it is shown in [20], for any $h>0$ general MIN FEEDBACK VERTEX SET is not in DGLO with respect to $h$-bounded neighborhoods; for any $k$ and any $h<k$, MAX $k$-SAT is not in DGLO with respect to $h$-bounded neighborhoods; B-KNAPSACK $\left(n^{k}\right)$ is not in 
DGLO with respect to $h$-bounded neighborhoods, for any $h>0$ (we have here a polynomial problem whose local optima do not have guaranteed quality); SUBSET SUM is not in DGLO with respect to $h$-bounded neighborhoods, for any $h>0$.

Let us now consider the relationship of DGLO with respect to the differential approximability class DAPX. Let

$$
\overline{\mathbf{G L O}}^{\text {PTAS }}=\left\{\Pi^{\prime}: \Pi^{\prime} \in \mathbf{N P O}, \exists \Pi \in \mathbf{G L O}, \Pi^{\prime} \leq_{\mathrm{PTAS}} \Pi\right\}
$$

be the closure of GLO under $\leq$ PTAS. Analogously, set

$$
\overline{\text { DGLO }}^{\text {DPTAS }}=\left\{\Pi^{\prime}: \Pi^{\prime} \in \mathbf{N P O}, \exists \Pi \in \mathbf{D G L O}, \Pi^{\prime} \leq \text { DPTAS } \Pi\right\}
$$

the closure of DGLO under $\leq_{\text {DPTAS }}$. In [7] it is proved that $\overline{\mathbf{G L O}}^{\text {PTAS }}=\mathbf{A P X}$. It is easy to show that the same holds for differential approximation.

Proposition 6. DAPX $=\overline{\boldsymbol{D} G L \boldsymbol{O}^{\mathrm{DPTAS}}}$.

Proof. Since DGLO $\subseteq$ DAPX and DPTAS-reduction preserves differential approximation ratios, then $\overline{\mathbf{D G L O}}^{\text {DPTAS }} \subseteq$ DAPX. On the other hand, since MAX INDEPENDENT SET- $B$ is DAPX-complete then for any problem $A \in$ DAPX, $A \leq$ DPTAS MAX INDEPENDENT SET- $B$. This last problem belongs to DGLO, hence DAPX $\subseteq \overline{\mathbf{D G L O}}^{\text {DPTAS }}$ and the proof of the proposition is complete.

Among other interesting properties of the class GLO, in [8] it is proved that MAX 3-SAT is complete in GLO $\cap$ MAX-SNP with respect to reductions that preserve the quality of local optima (called LOP-reduction; see Definition 3 in Section 2). A related result in [18] shows that MAX-SNP $\subseteq$ Non-Oblivious GLO, a variant of the class GLO defined by means of local search algorithms that are allowed to use more general kinds of objective functions, rather than the natural objective function of the given problem, for improving the quality of the solution (note that by making use of the LOP-reduction to MAX 3-SAT, presented in [8], all problems in MAX-SNP can be solved by non-oblivious local search up to a solution of guaranteed quality). Such result it is indeed based upon the existence of a MAX-SNP problem (MAX $k$-CSP) which is general enough that all MAX-SNP problems can be formulated as subclasses of it and which therefore plays again the role of a complete problem.

In what follows, we show the existence of complete problems for a large, natural subclass of DGLO. As one can see from Definition 3 in Section 2, the local optimality preserving properties do not depend on the approximation measure adopted. Hence, in an analogous way, we define here a reduction called DLOP which is a DPTAS-one with the same local optimality preserving properties as the ones in Definition 3.

Definition 9. A DLOP-reduction is a DPTAS-reduction with the same surjectivity, partial monotonicity, locality and dominance properties as an LOP-reduction.

Proposition 7. Given two NPO problems $\Pi$ and $\Pi^{\prime}$, if $\Pi \leq{ }_{\mathrm{DLOP}} \Pi^{\prime}$ and $\Pi^{\prime} \in \boldsymbol{D G} \boldsymbol{G} \boldsymbol{O}$, then $\Pi \in \boldsymbol{D G L O}$.

Let DGLO $_{0}$ be the class of MAX-SNP maximization problems that belong to DGLO and for which the worst value 0 is feasible for any instance (MAX INDEPENDENT SET- $B$, for example, is such a problem). Note that for the problems of $\mathbf{D G L O}_{\mathbf{0}}$, the standard and differential approximation ratios coincide. Now let us consider the closure of $\mathbf{D G L O}_{\mathbf{0}}$ under affine transformations. This leads to the following definition. 
Definition 10. Let $\Pi$ be a polynomially bounded NPO problem. Then, $\Pi \in$ DGLO $^{\prime}$ if (i) it belongs to $\mathbf{D G L O}_{0}$, or(ii) it can be transformed into a problem in $\mathbf{D G L O}_{\mathbf{0}}$ by means of an affine transformation; in other words, $\mathbf{D G L O}^{\prime}={\overline{\mathbf{D G L O}_{0}}}^{\mathbf{A F}}$.

Now we can prove the following result.

Theorem 7. For any problem $\Pi \in$ DGLO' $^{\prime} \Pi \leq$ DLop MAX IndEPEndent SET- $B$.

Proof. Assume $\Pi \in \mathbf{D G L O}^{\prime}$. We then have the following two cases: (i) $\Pi \in \mathbf{D G L O}_{\mathbf{0}}$ or (ii) can be transformed into a problem in $\mathbf{D G L O}_{\mathbf{0}}$ by means of an affine transformation.

We first deal with case (i), i.e., we consider $\Pi \in \mathbf{D G L O}_{\mathbf{0}}$. Then, the following assertions hold:

- $\Pi \in \mathbf{D G L O}_{\mathbf{0}}$ implies $\Pi \in \mathbf{G L O}$ (by the way $\mathbf{D G L O} \mathbf{O}_{\mathbf{0}}$ has been defined just above);

- $\Pi \in \mathbf{G L O}$ implies $\Pi \in$ MAX-SNP (by the definition of GLO);

- by the result of [8]:

$$
\Pi \leq \text { LOP MAX 3-SAT }
$$

Observe next that the L-reduction of [23] between MAX 3-SAT and MAX 3-SAT- $B$, transforms any feasible solution for MAX 3-SAT- $B$ of value $m$ to a solution for MAX 3-SAT of value $m^{\prime}=m-K$ where $K$ is a function of $c$ (the rate of the expander used) and of the optimal value of the instance of MAX 3-SAT. Hence, the L-reduction of MAX 3-SAT to MAX 3-SAT-B is an LOP-one:

$$
\text { MAX 3-SAT } \leq \text { LOP MAX 3-SAT- } B
$$

Finally, remark that the L-reduction of [23] between MAX 3-SAT- $B$ and MAX INDEPENDENT SET- $B$ is also an LOP-one since it preserves equality of feasible values:

$$
\text { MAX } 3 \text {-SAT- } B \leq \text { LOP MAX INDEPENDENT SET- } B
$$

From (9), (10) and (11), and since LOP-reductions are transitive, one concludes that for any $\Pi \in \mathbf{D G L O}_{\mathbf{0}}, \Pi \leq$ LoP MAX INDEPENDENT SET- $B$. Finally, it is easy to see that for $\mathbf{D G L O}_{\mathbf{0}}$, an LOP-reduction is also a DLOP-one.

We now deal with case (ii), i.e., we assume that $\Pi$ is obtained by means of an affine transformation from a problem $\Pi^{\prime} \in \mathbf{D G L O}_{\mathbf{0}}$. Since an affine transformation is a DLOP-reduction,

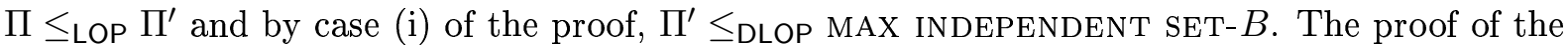
theorem is then completed.

Proposition 8. MAX CUt, Min Vertex COVER- $B$, MAX Set PACKING- $B$, Min SET Cover- $B$ are $\boldsymbol{D} \boldsymbol{G L} \boldsymbol{O}^{\prime}$-complete, under DLOP-reductions.

Proof. The result for MAX CUT and MAX SET PACKING- $B$ follows from the fact that both problems belong to $\mathbf{D G L O}_{0}$ and from Theorem 7. The result for MIN VERTEX COVER- $B$ follows from the fact that MAX INDEPENDENT SET is affinely transformable to it. Finally, the result for MIN SET COVER- $B$ follows from the fact that it is a generalization of MIN VERTEX COVER- $B$.

In all, Figure 3 summarizes the image of the several classes discussed in this section and of their relationships. Note that MIN MULTIPROCESSOR SCHEDULING, or even MIN and MAX TSP on graphs with polynomially bounded edge-distances belong to DGLO ([20, 21]) but neither to GLO, nor to DGLO'. On the other hand, MIN VERTEX COver- $B$ belongs to DGLO' but not to MAX-SNP. 


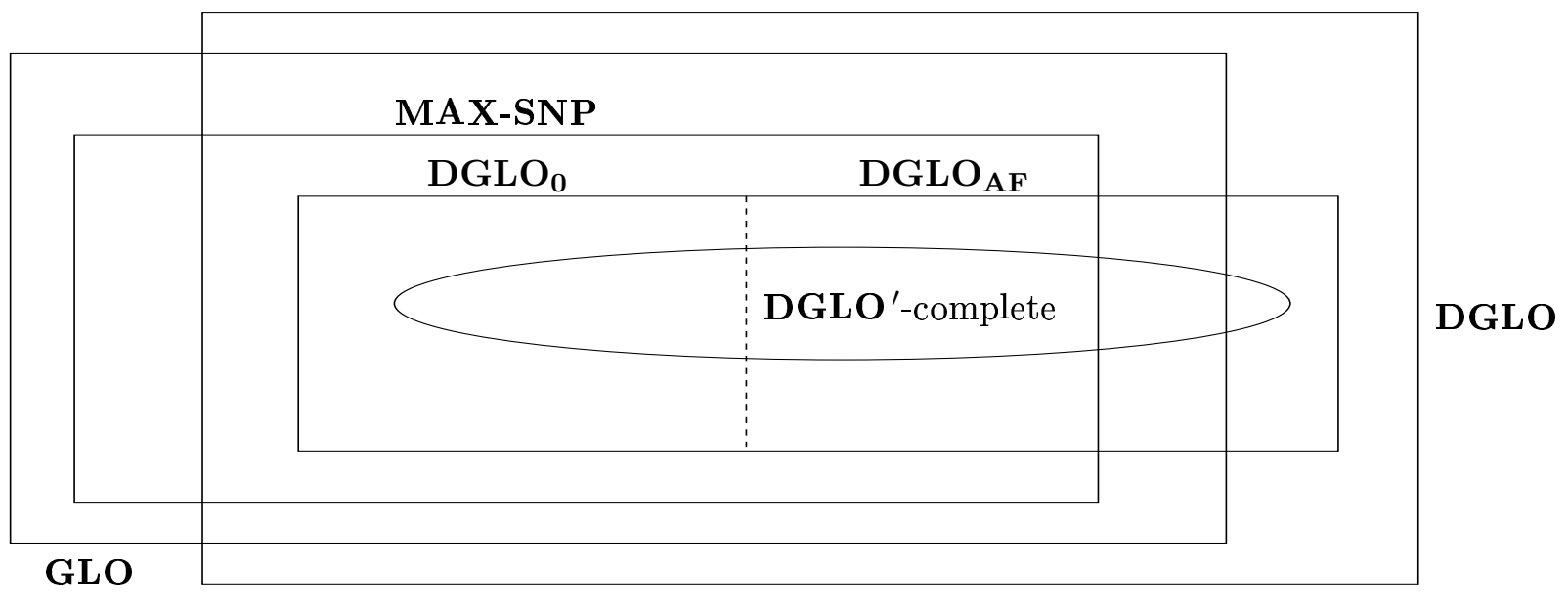

Figure 3: GLO, DGLO, MAX-SNP, $\mathbf{D G L O}^{\prime}=\mathbf{D G L O}_{\mathbf{0}} \cup \mathbf{D G L O}_{\mathrm{AF}}, \mathbf{D G L O}^{\prime}$-complete.

\section{Concluding remarks}

We have proposed a structure for differential approximability classes. One of the interesting points in this work is that the results obtained confirm our intuition that differential approximation is rich enough to motivate further both "structural" and "computational" research. We have defined natural reductions and proved completeness of natural NPO problems for DAPX and for a subclass of DGLO. Moreover, we have exhibited the hard problems for DPTAS without, unfortunately, providing complete problems for it. The existence of such problems is an interesting open problem. Another point for further investigation is the existence of intermediate problems in differential approximability classes, i.e., problems which, unless $\mathbf{P}=\mathbf{N P}$, or another complexity theoretic hypothesis, they belong to a certain approximability class and they are neither complete for it, nor belong to an inferior class.

\section{References}

[1] A. Aiello, E. Burattini, M. Furnari, A. Massarotti, and F. Ventriglia. Computational complexity: the problem of approximation. In C. M. S. J. Bolyai, editor, Algebra, combinatorics, and logic in computer science, volume I, pages 51-62, New York, 1986. North-Holland.

[2] G. Ausiello, P. Crescenzi, G. Gambosi, V. Kann, A. Marchetti-Spaccamela, and M. Protasi. Complexity and approximation. Combinatorial optimization problems and their approximability properties. Springer, Berlin, 1999.

[3] G. Ausiello, P. Crescenzi, and M. Protasi. Approximate solutions of NP optimization problems. Theoret. Comput. Sci., 150:1-55, 1995.

[4] G. Ausiello, A. D'Atri, and M. Protasi. On the structure of combinatorial problems and structure preserving reductions. In Proc. ICALP'77, Lecture Notes in Computer Science. Springer-Verlag, 1977.

[5] G. Ausiello, A. D'Atri, and M. Protasi. Structure preserving reductions among convex optimization problems. J. Comput. System Sci., 21:136-153, 1980.

[6] G. Ausiello, A. Marchetti-Spaccamela, and M. Protasi. Towards a unified approach for the classification of NP-complete optimization problems. Theoret. Comput. Sci., 12:83-96, 1980. 
[7] G. Ausiello and M. Protasi. Local search, reducibility and approximability of NPoptimization problems. Inform. Process. Lett., 54(2):73-79, 1995.

[8] G. Ausiello and M. Protasi. NP optimization problems and local optima graph theory. In Y. Alavi and A. Schwenk, editors, Combinatorics and applications. Proc. 7th Quadriennal International Conference on the Theory and Applications of Graphs, volume 2, pages 957975, 1995.

[9] C. Bazgan and V. T. Paschos. Differential approximation for optimal satisfiability and related problems. European J. Oper. Res., 147(2):397-404.

[10] S. A. Cook. The complexity of theorem-proving procedures. In Proc. STOC'71, pages 151-158, 1971.

[11] P. Crescenzi, V. Kann, R. Silvestri, and L. Trevisan. Structure in approximation classes. SIAM J. Comput., 28(5):1759-1782, 1999.

[12] P. Crescenzi and A. Panconesi. Completeness in approximation classes. Inform. and Comput., 93(2):241-262, 1991.

[13] P. Crescenzi and L. Trevisan. On approximation scheme preserving reducibility and its applications. In Foundations of Software Technology and Theoretical Computer Science, FCT-TCS, number 880 in Lecture Notes in Computer Science, pages 330-341. SpringerVerlag, 1994.

[14] M. Demange, P. Grisoni, and V. T. Paschos. Differential approximation algorithms for some combinatorial optimization problems. Theoret. Comput. Sci., 209:107-122, 1998.

[15] M. Demange and V. T. Paschos. Quelques étapes vers la conciliation de la théorie d'approximation et celle d'optimisation : une nouvelle théorie d'approximation polynomiale et résultats préliminaites. C. R. Acad. Sci. Paris Sér. I Math., 317:409-414, 1993.

[16] M. Demange and V. T. Paschos. On an approximation measure founded on the links between optimization and polynomial approximation theory. Theoret. Comput. Sci., 158:117-141, 1996.

[17] R. Hassin and S. Khuller. z-approximations. jalgo, 41:429-442, 2001.

[18] S. Khanna, R. Motwani, M. Sudan, and U. Vazirani. On syntactic versus computational views of approximability. SIAM J. Comput., 28:164-191, 1998.

[19] J. Monnot. Differential approximation results for the traveling salesman and related problems. Inform. Process. Lett., 82(5):229-235, 2002.

[20] J. Monnot, V. T. Paschos, and S. Toulouse. Optima locaux garantis pour l'approximation différentielle. Technical report, LAMSADE, Université Paris-Dauphine, 2002. In french. Available on http://www.lamsade.dauphine.fr/cahdoc.html\#cahiers.

[21] J. Monnot, V. T. Paschos, and S. Toulouse. Approximation algorithms for the traveling salesman problem. Mathematical Methods of Operations Research, 57(1):387-405, 2003.

[22] P. Orponen and H. Mannila. On approximation preserving reductions: complete problems and robust measures. Technical Report C-1987-28, Dept. of Computer Science, University of Helsinki, Finland, 1987. 
[23] C. H. Papadimitriou and M. Yannakakis. Optimization, approximation and complexity classes. J. Comput. System Sci., 43:425-440, 1991.

[24] S. Toulouse. Approximation polynomiale: optima locaux et rapport différentiel. $\mathrm{PhD}$ thesis, LAMSADE, Université Paris-Dauphine, 2001. In French.

[25] E. Zemel. Measuring the quality of approximate solutions to zero-one programming problems. Math. Oper. Res., 6:319-332, 1981.

\section{A A list of NPO problems}

We present the list of NPO problems mentioned and/or discussed in the paper, together with a characterization of their worst-value solutions. For most of these problems, comments about their approximability in standard approximation can be found in [2]. MAX WSAT- $B$ and MAX LINEAR WSAT- $B$ are also dealt in [3, 12], respectively.

\section{Maximum and minimum weighted satisfiability (MAX WSAT and MIN WSAT).}

Given a boolean formula $\varphi$ with non-negative integer weights $w(x)$ on any variable $x$ appearing in $\varphi$, MAX WSAT consists of computing a truth assignment to the variables of $\varphi$ that both satisfies $\varphi$ and maximizes the sum of the weights of the variables set to 1 . We consider that the assignment setting all the variables to 0 , even if it does not satisfy $\varphi$, is feasible and represents the worst-value solution for MAX WSAT. In MIN WSAT, the objective is to minimize the total weight of an assignment satisfying $\varphi$. Here we assume the assignment setting any variable to 1 to be feasible (even if it does not satisfy $\varphi$ ) and to represent the worst-value solution for MIN WSAT. By MAX WSAT- $B$, we denote the version of MAX WSAT where the variable-weights are polynomially bounded and their sum lies in the interval $[B, 2 B]$. By MAX w $k$-SAT, we denote the version of MAX WSAT where no clause contains more than $k$ literals. Finally, by MAX LINEAR WSAT- $B$ we will denote the version of MAX WSAT where the variable-weights are polynomially bounded and their sum lies in the interval $[B,(n /(n-1)) B]$. For both MAX WSAT- $B$ and MAX LINEAR WSAT- $B$ it is assumed that the assignment setting all variables to 0 is feasible and that its value is $B$. Obviously, this assignment represents the worst feasible value for these problems.

Maximum and minimum $\{0,1\}$ integer programming (MAX $\{0,1\}$ INTEGER PROGRAMMING and MIN $\{0,1\}$ INTEGER PROGRAMMING).

Given an integer matrix $A$, an integer vector $\vec{b}$ and a positive integer vector $\vec{c}$, the objective is to determine a $\{0,1\}$-vector $\vec{x}$ satisfying $A \cdot \vec{x} \leqslant \vec{b}$ and maximizing $\vec{c} \cdot \vec{x}$ (for MAX $\{0,1\}$ Integer PROGRAMMing), or satisfying $A \cdot \vec{x} \geqslant \vec{b}$ and minimizing $\vec{c} \cdot \vec{x}$ (for MiN $\{0,1\}$ Integer PROGRAmming). Worst-value solutions here are vectors $\{0\}^{n}$ and $\{1\}^{n}$, respectively.

Maximum satisfiability (MAX SAT).

Given a boolean CNF $\varphi$, the objective is to compute a truth assignment to the variables maximizing the number of clauses satisfied. By MAX $k$-SAT, we denote the version of MAX SAT where no clause contains more than $k$ literals. Worst-value solution: the optimal solution of MIN SAT (where we wish to compute a truth assignment to the variables minimizing the number of clauses satisfied) on $\varphi$.

Maximum independent set (MAX INDEPENDENT SET).

Given a graph $G(V, E)$, an independent set is a subset $V^{\prime} \subseteq V$ such that whenever 
$\left\{v_{i}, v_{j}\right\} \subseteq V^{\prime}, v_{i} v_{j} \notin E$, and MAX INDEPENDENT SET consists in finding an independent set of maximum size. By MAX INDEPENDENT SET- $B$, we denote MAX INDEPENDENT SET in bounded-degree graphs. Worst-value solution: the empty set.

Maximum clique (MAX CLIQUE).

Consider a graph $G(V, E)$. A clique of $G$ is a subset $V^{\prime} \subseteq V$ such that every pair of vertices of $V^{\prime}$ are linked by an edge in $E$, and MAX CLIQUE consists in finding a maximum size set $V^{\prime}$ inducing a clique in $G$ (a maximum-size clique). Worst-value solution: the empty set.

\section{Minimum coloring (MIN COLORING).}

Given a graph $G(V, E)$, we wish to color $V$ with as few colors as possible so that no two adjacent vertices receive the same color. Worst-value solution: $V$.

Maximum $\ell$-colorable induced subgraph (MAX $\ell$-COLORABLE INDUCED SUBGRAPH). Given $\ell<\Delta(G)$ (the maximum graph-degree), MAX $\ell$-COLORABLE INDUCED SUBGRAPH consists of finding, in a graph $G(V, E)$, a maximum-order subgraph $G^{\prime}$ of $G$ that is $\ell$ colorable. Worst-value solution: the empty set.

Minimum vertex-covering (MIN VERTEX COVER).

Given a graph $G(V, E)$, a vertex cover is a subset $V^{\prime} \subseteq V$ such that, $\forall u v \in E$, either $u \in V^{\prime}$, or $v \in V^{\prime}$, and MIN VERTEX COVER consists of determining a minimum-size vertex cover. Worst-value solution: $V$.

\section{Minimum set-covering (MIN SET COVER).}

Given a collection $\mathcal{S}$ of subsets of a finite set $C$, a set cover is a sub-collection $\mathcal{S}^{\prime} \subseteq \mathcal{S}$ such that $\cup_{S_{i} \in \mathcal{S}^{\prime}} S_{i}=C$, and MIN SET COVER consists of finding a cover of minimum size. We denote by MIN SET COVER- $B$ the restriction of MIN SET COVER on set-systems where $\left|S_{i}\right| \leqslant B, S_{i} \in \mathcal{S}^{\prime}$. Also, we denote by MIN SET w $(K)$ COVER- $B$, an instance of MIN SET COVER- $B$ where, given a fixed constant $K$, integer weights less than, or equal to, $K$ are assigned to the sets of $\mathcal{S}$ and the objective consists of minimizing the total weight of a set cover. Worst-value solution for MIN SET COVER: $\min \{|\mathcal{S}|,|C|\}$ and for its weighted version the total weight of $\mathcal{S}$.

\section{Maximum set-packing (MAX SET PACKING).}

Given a collection $\mathcal{S}$ of subsets of a finite set $C$, a set packing is a sub-collection of mutually disjoint sets of $\mathcal{S}^{\prime}$ and MAX SET PACKING consists of determining a set packing of maximum size. We denote by MAX SET PACKING- $B$ the restriction of MAX SET-PACKING on setsystems where $\left|S_{i}\right| \leqslant B, S_{i} \in \mathcal{S}^{\prime}$. Worst-value solution: the empty set.

Minimum dominating set (MIN DOMINATING SET).

Given a graph $G(V, E)$, the objective is to compute a subset $V^{\prime} \subseteq V$ such that $\forall u \in V \backslash V^{\prime}$, there exists $v \in V^{\prime}$ for which $u v \in E$. By Min DOMinating SET- $B$, we denote MiN DOMINATING SET in bounded-degree graphs. Worst-value solution: $V$.

Minimum independent dominating set (MIN INDEPENDENT DOMINATING SET). Given a graph $G(V, E)$, the objective is to compute a maximal (for the inclusion) independent set of minimum size. Worst-value solution: a maximum independent set.

\section{Maximum cut (MAX CUT).}

Given a graph $G(V, E)$, the objective is to determine a subset $V^{\prime} \subseteq V$ maximizing the number of edges whose one endpoint is in $V^{\prime}$ and the other one in $V \backslash V^{\prime}$. Worst-value solution: the empty set. 
Minimum feedback edge set (MIN FEEDBACK EDGE SET).

Given a graph $G(V, E)$, the objective is to determine a minimum size subset $E^{\prime} \subseteq E$ such that any cycle in $G$ uses at least one edge in $E^{\prime}$. Worst-value solution: $E$.

\section{Minimum feedback vertex set (MIN FEEDBACK VERTEX SET).}

Given a graph $G(V, E)$, the objective is to determine a minimum size subset $V^{\prime} \subseteq V$ such that any cycle in $G$ uses at least one vertex in $V^{\prime}$. By MIN FEEDBACK VERTEX SET- $B$, we denote the version of MIN FEEDBACK VERTEX SET in bounded-degree graphs. Worst-value solution: $V$.

\section{Minimum multiprocessor scheduling (MIN MULTPROCESSOR SCHEDULING).}

Given $n$ tasks $T_{1}, \ldots, T_{n}$ together with their respective execution lengths $\ell_{1}, \ldots, \ell_{n}$, we search for allocating the tasks to $m$ processors in such a way that the overall deadline of the most used processor is minimized (there are no precedence constraints). We assume that execution lengths are bounded by a polynomial of $n$. Worst-value solution is the one where one allocates all the tasks to only one processor; the value of such solution is $\sum_{i=1}^{n} \ell_{i}$.

\section{Binary knapsack (B-KNAPSACK).}

Given two integer $n$-vectors $\vec{a}$ and $\vec{b}$ and an integer $B$, the objective is to determine a vector $\vec{x} \in\{0,1\}^{n}$ which, under the constraint $\vec{b} \cdot \vec{x} \leqslant B$, maximizes the scalar $\vec{a} \cdot \vec{x}$. We denote by B-KNAPSACK $\left(n^{k}\right)$, the restriction of B-KNAPSACK in instances where the greatest number is at most $n^{k}$. Remark that B-KNAPSACK $\left(n^{k}\right) \in \mathbf{P}$. Worst-value solution: the vector $\{0\}^{n}$.

\section{Subset sum (sUBSET SUM).}

This is as B-KNAPSACK with the additional constraint that vectors $\vec{a}$ and $\vec{b}$ coincide. Worstvalue solution: the vector $\{0\}^{n}$.

\section{Minimum and maximum traveling salesman problem (MIN and MAX TSP).}

Given a complete graph on $n$ vertices, denoted by $K_{n}$, with positive distances on its edges, MIN TSP (resp., MAX TSP) consists of minimizing (resp., maximizing) the cost of a Hamiltonian cycle (an ordering $\left\langle v_{1}, v_{2}, \ldots, v_{n}\right\rangle$ of $V$ such that $v_{n} v_{1} \in E$ and, for $1 \leqslant i<n$, $\left.v_{i} v_{i+1} \in E\right)$, the cost of such a cycle being the sum of the distances of its edges. Worstvalue solution of the former is an optimal solution for the latter in the same instance, and vice-versa.

Note that MAX INDEPENDENT SET, MAX SET PACKING and MAX CLIQUE are approximate equivalent under both standard and differential approximation ratios; moreover, the two ratios coincide for each of these problems. For MAX $\ell$-COLORABLE INDUCED SUBGRAPH standard and differential approximation ratios coincide. Furthermore, the differential ratio for MIN VERTEX COVER coincides with the standard and differential ratios for MAX INDEPENDENT SET. 